Z. klin. Chem. u. klin. Biochem.

8. Jg., S. $288-292$, Mai 1970

\title{
Über die Glykoproteine, Plasmaproteine und Glykosaminoglykane in Normalurin
}

\author{
Von D. GräsSLIN $\left.{ }^{1}\right)$, H. WeICKer und D. BarWICH \\ S. J. Thannbauser Abteilung fïr Stofivecbseluntersucbungen (Leiter: Prof. Dr. H. Weicker) der Medizinischen Universitäts- \\ poliklinik Heidelberg (Kommiss. Direktor: Prof. Dr. E. Kubn)
}

(Eingegangen am 12. Dezember 1969)

Der Mittelwert für die physiologische Proteinurie liegt für Normalpersonen bei $170 \mathrm{mg} / 24 \mathrm{Stdn}$. mit einer Variationsbreite von 150 bis $310 \mathrm{mg} / 24 \mathrm{Stdn}$. Zwischen Männern und Frauen und auch innerhalb des untersuchten Altersbereichs von 20 bis 40 Jahren wurden keine unterschiedlichen Werte gefunden.

Das nach Druckfiltration, Dialyse und Lyophilisation gewonnene Substanzgemisch enthält' etwa 40\% Kohlenhydrate, davon etwa $10 \%$ N-Acetylneuraminsäure. Der undialysierbare Urinanteil besteht zu rund $10 \%$ aus Plasmaproteinen, zu $10 \%$ aus Glykosaminoglykanen und zu 10\% aus anorganischen Bestandteilen. Die Kohlenhydratkomponenten wurden chromatographisch dargestellt und das molare Verhältnis der Neutralzucker sowie die prozentuale Verteilung ermittelt. Das Substanzgemisch wurde diskelektrophoretisch aufgetrennt, die Plasmaproteine mit Amidoschwarz und die Glykosaminoglykane mit Alcianblau dargestellt.

\section{Glycoprottins, plasma proteins and glycosaminoglycans in normal urine}

The average ivalue for physiological proteinuria in normal persons is $170 \mathrm{mg} / 24 \mathrm{hr}$., with a range of variation of $150-310 \mathrm{mg} / 24 \mathrm{hr}$. All values fell within this range for the age group $20-40$ years and for both sexes.

After pressure filtration, dialysis and lyophilisation, the resulting mixture contains about $40 \%$ carbohydrate, of which $10 \%$ is $\mathrm{N}-a$ cetylneuraminic acid. The undialysable urine fraction consists of about $10 \%$ plasma proteins, $10 \%$ glycosaminoglycans and $10 \%$ inorganic material. The carbohydrate components were separated chromatographically and the molar proportions of neutral sugars and their percentage distribution wete determined. The mixture was separated by disc electrophoresis, the plasma proteins stained with amido black and the glycosaminoglycans with alcian blue.

MörNer (1) wies bereits 1895 im Normalurin des Menschen Proteine nach. Wie wir heute wissen, bestehen der nicht dialysierbare und der nicht ultrafiltrierbare Anteil des Normalurins aus einem Gemisch von Glykoproteinen, Plasmaproteinen und sauren Mucopolysacchariden (Glykosaminoglykanen). Die Molekulargewichte dieses sehr heterogenen Substanzgemisches schwanken zwischen mehreren Tausend (2) und einigen Millionen (3). Die Angaben in der Literatur über die Eiweißkonzentrationen im Urin variieren noch erheblich (4).

Das sog. TAMm-Horsfall-Mucoid (5), identisch mit der "Nubecula“, konnte bisher als einzige großmolekulare Eiweißfraktion in reiner Form aus Urin isoliert, in seiner Zusammensetzung und seinen physikochemischen Eigenschaften genauer charakterisiert werden $(6,7)$. Das Molgewicht dieses Glykoproteins beträgt $28 \cdot 10^{6}$; es kann in Untereinheiten bis herunter zum Molekulargewicht $1,7 \cdot 10^{6}$ dissoziieren, wie MAxFIELD und Mitarbeiter (8) zeigen konnten und ist dann identisch mit dem von dr Ferrante und Rich (9) isolierten Glykoprotein.

HAMERMAN (10) und KING Jr. und Mitarbeiter (11) stellten später zuerst fest, daß der nichtdialysierbare Anteil des Normalurins zum größten Teil aus relativ niedermolekularen Glykoproteinen mit hohem Kohlenhydratgehalt besteht. Allerdings gelang es bis jetzt nicht, eines dieser Glykoproteine darzustellen.

1) Jetzige Anschrift: Universitäts-Frauenklinik, Hamburg, Hormonlabor.
Mit Hilfe der Immunoelektrophorese hat man sich heute einen Überblick verschafft über das Plasmaproteinspektrum des Normalurins. Vor rund zehn Jahren wurden mit dieser Methode zuerst von GRANT (12), Patte (13) und Biserte (14) die Anwesenheit einzelner Serumproteine gesichert. In den letzten Jahren erschienen hierzu zahlreiche Arbeiten (15-19), so daß heute insgesamt 19 verschiedene serumidentische Proteine in Normalurin nachgewiesen werden konnten. Albumin reptäsentiert mit etwa $70 \%$ den Hauptanteil an ausgeschiedenen Plasmaproteinen (20).

Die dritte Gruppe nichtdialysierbarer Substanzen umfaßt die Glykosaminoglykane, auf deren Ausscheidung schon sehr früh hingewiesen wurde (21-23); jedoch war die chemische Klassifizierung noch unzureichend. Dagegen gelang. KERBY (24) 1954 die Isolierung von Glykosaminoglykanen aus Urin von Normalpersonen, deren Hauptkomponente Chondroitinsulfate waren. Nach zahlreichen weiteren Untersuchungen (25-32) ist inzwischen die Differenzierung in Einzelkomponenten weitgehend gelungen, wenn auch die quantitative Seite je nach Untersuchungsmethode noch größeren Schwankungen unterworfen ist. Es ist noch nichts bekannt über die Molekulargewichte der ausgeschiedenen Glykosaminoglykane und auch nicht darüber, ob sie kovalent gebundene Polypeptidanteile besitzen.

In der vorliegenden Arbeit wurde der gesamte nichtdialysierbare Antèil des Normalurins von Männern und Frauen im Alter $z$ wischen 20 und 40 Jahren quantitativ 
erfaßt und durch Bestimmung der Neutralzucker, Aminozucker, Hexuronsäuren, des N-Gehaltes und der anorganischen Bestandteile charakterisiert. Außerdem wurden die in Glykoproteinen und Glykosaminoglykanen vorliegenden Neutralzucker nach saurer $\mathrm{Hy}$ drolyse chromatographisch dargestellt und das molare Verhältnis bestimmt.

Mit Hilfe der Polyacrylamidgel-Elektrophorese und aus den Analysendaten wurde versucht, Aufschluß zu erhalten über die Zusammensetzung des Substanzgemisches.

\section{Material und Aufarbeitung}

Achtzehn 24-Stdn.-Urine wurden von 15 klinisch gesunden Personen (5 Frauen, 10 Männer) im Alter von 20 bis 40 Jahren gesammelt. Als Schutz gegen bakterielles Wachstum wurde Natriumazid $(10 \mathrm{mg} / 100 \mathrm{ml})$ in die Polyäthylenflaschen zugegeben. Die Proben wurden mit der Essigsäure-Kochprobe und dem Combistix-Test auf Anwesenheit von Protein und Glucose unter sucht; alle Teste fielen negativ aus.

Nach $15 \mathrm{Min}$. Zentrifugation der gesamten festgelegten Urinmenge bei 3500 U./Min. wurden Sediment und Zelldetritus verworfen und der Uberstand zur Abtrennung submikroskopischer Partikel druckfiltriert. Druckfiltrationsgerät Typ SM $16208 \mathrm{ml}$ Fassungsvermögen (Fa. Sartorius Membranfilter, Göttingen, Deutschland). Als Membranfilter diente der Filtertyp SM 11007 (Material: Cellulosenitrat) mit mittlerem Porendurchmesser von $0,25 \mu \mathrm{m}$; Druck bis 8 atü.

Anschließend Dialyse in Visking-Schläuchen ${ }^{2}$ ) (23/32), zuerst $48 \mathrm{Stdn}$. gegen einen kräftigen Strom fließenden Wassers, dann $24 \mathrm{Stdn}$. gegen $30 l$ dest. Wassers von $4^{\circ}$, das durch ein Rührwerk in Bewegung gehalten wurde.

Gefriertrocknung des Dialyserückstandes ergibt Substanz A. Nach Druckfiltration über Filter SM 11007, und Lyophilisation des Filtrates einer 1proz. wäßr. Lösung von A exhält man B. Zur Gewinnung von $C$ wurde eine $500 \mathrm{mg}$ proz. wäßr. Lösung ( $500 \mathrm{mg} /$ $100 \mathrm{ml}$ ) von B $72 \mathrm{Stdn}$. gegen $1 /$ dest. Wasser bei $4^{\circ}$ dialysiert. Das Wasser wurde durch starkes Magnetrühren in Bewegung gehalten, das Dialysat mehrmals gewechselt. Gefriertrocknung von Schlauchinhalt ergibt Substanz C. Alle Substanzen wurden für die Analysen in vacuo bei $50^{\circ}$ über $\mathrm{P}_{4} \mathrm{O}_{11}$ bis zur Gewichtskonstanz getrocknet.

\section{Methoden}

Diskontinuierliche Polyacrylamidgel-Elektrophorese nach MAURER (33), ausgeführt mit Disc-Elektrophoresegerät $\mathrm{Fa}$. Shandon (London). Gelsystem Nr. $\mathrm{I}_{34}$, pH 8,9, 7,5proz. bzw. 5proz. Gel. Elektrodenpuffer: Tris-Glycin, $\mathrm{pH} 8,3$.

Anfärbung der Gelzylinder:

Amidoschwarz 10 B (1proz. Amidoschwarz in 7proz. wäßrigmethanolischer Essigsäure) für Proteine und Glykoproteine.

Alcianblau (2proz. Alcianblạu in 2proz. Essigsäure (35)) für Glykosaminoglykane.

$P A S-F$ ärbung für Glykoproteine nach KAO (36).

Agar-Immunoelektrophorese in det Modifikation nach ScHEIDEGGER (36a).

Dïnnschichtchromatographie, Papierchromatographie und Bestimmung der Neutralzucker mit Triphenyl-tetrazoliumchlorid nach den von uns bereits mitgeteilten Verfahren (37).

Analysen und Bestimmungsmethoden

Gesamtneuraminsäure nach SVENNERHOLM (38).

Enzymatisch bestimmbare Neuraminsäure nach WARREN (39). Neutralzucker mit Anthron (40) - und Orcin-Methode (41).

2) Die Dialysemembran hält globuläre Proteine zurück bis herunter zum Molekulargewicht von 12-15000.
Fucose nach Dische (42).

Hexuronsäuren mit Carbazol-Methode nach Dische (43), in der Modifikation nach GREGORY (44).

Aminozucker nach Cessi (45).

Proteingebalt nach KJELDari-Methode (46).

Anorganisclse Bestandteile nach Verglühen der Substanz bei $800^{\circ}$ : flammenphotometrische Bestimmung von $\mathrm{Na}^{+}, \mathrm{K}^{+}, \mathrm{Ca}^{++}$.

\section{Ergebnisse}

Die Menge des in $24 \mathrm{Stdn}$. ausgeschiedenen nichtdialysierbaren Anteils in Normalurin (gewonnen über Druckfiltration, Dialyse und Gefriertrocknung) beträgt $150-200 \mathrm{mg}$. Nur bei zwei von insgesamt 15 untersuchten Normalpersonen liegt die Ausscheidungsrate mit $280 \mathrm{mg}$ bzw. $310 \mathrm{mg}$ oberhalb des gefundenen Bereichs. Die mittlere Ausscheidungsmenge an Substanz A liegt bei $170 \mathrm{mg} / 24 \mathrm{Stdn}$, wobei unterschiedliche Urinmengen pro $24 \mathrm{Stdn}$. (zwischen 480 und $1800 \mathrm{ml}$ ) hierbei keine Rolle spielen.

Tabelle 1 zeigt die Analysenergebnisse der isolierten Substanzen A, B und C (siehe „Material und Aufarbeitung ") von 15 Normalpersonen beiderlei $\mathrm{Ge}-$ schlechts im Alter zwischen 20 und 40 Jahren.

Der mittlere Gesamtkohlenhydratgehalt des nichtdialysierbaren Urinanteils liegt für Substanz A bei $40,5 \%$, steigt für den wäßr. Auszug von A (Substanz B) auf $44,8 \%$ an und erreicht nach nochmaliger Dialyse (Sub$\operatorname{stanz}$ C) einen Mittelwert von 51,4\%. Die entsprechenden Zunahmen der einzelnen Kohlenhydratbestandteile von Substanz A über B nach $C$ ist aus Tabelle 1 ersichtlich.

Es treten sowohl in den Analysenergebnissen als auch in der Menge des nichtdialysierbaren Anteils von Normalurin zwischen Männern und Frauen keine signifikanten Unterschiede auf. In bezug auf diese Werte bestand bei dem vorgelegten Untersuchungsgut auch keine Altersabhängigkeit.

Mit Hilfe der Dünnschichtchromatographie konnten die bereits bekannten Zucker (48) Galaktose, Mannose, Fucose und geringe Mengen an Xylose als Bestandteile der Glykoproteine und Glykosaminoglykane des Dialyserückstandes dargestellt werden (Abb. 1). Das Verhältnis der einzelnen Monosaccharide wurde für Substanz A nach papierchromatographischer Auftrennung mit der Triphenyltetrazolium-Methode (49) wie folgt ermittelt:

$$
\text { Gal:Man:Fuc: } \mathrm{Xyl}=9: 4: 2,5: 0,1 \text {. }
$$

Der Xylose-Gehalt steigt von A nach $C$ leicht an.

Für die Aminozucker wurde papierchromatographisch nach Darstellung mit alkalischer Silbernitratlösung und densitometrischer Auswertung ein Verhältnis von Glucosamin: Galaktosamin $=2: 1$ gefunden.

Die Substanzgemische $A$ und $C$ wurden mit Hilfe der Polyacrylamidgelelektrophorese aufgetrennt (Abb. 2). Gelzylinder G 1 zeigt Normalserum, G 2 Substanz A, G 3 Substanz C, angefärbt mit Amidoschwarz $10 \mathrm{~B}$. Von den sechs dargestellten Fraktionen der Substanz A konnte die stärkste Bande als Albumin, die elektrophoretisch schneller als Albumin wandernde Zone als 
Tab. 1

Analysenergebnisse der nichtdialysierbaren Anteile in Normalurin

(in Prozent, bezogen auf $\mathrm{mg}$ Trockengewicht; Normalbereiche und Mittelwerte)

\begin{tabular}{|c|c|c|c|c|c|c|c|c|c|c|c|}
\hline $\begin{array}{l}\text { Sub- } \\
\text { stanz }\end{array}$ & $\begin{array}{c}\mathrm{N}-\text { Acetyl- } \\
\text { neuraminsäure }\end{array}$ & Fucose & $\begin{array}{l}\text { Hexosen } \\
\text { (Anthron) }\end{array}$ & $\begin{array}{c}\text { Hexosen } \\
\text { (Orcin) }\end{array}$ & $\begin{array}{l}\text { Hexos- } \\
\text { amin }\end{array}$ & $\begin{array}{l}\text { Hexuron- } \\
\text { säure }\end{array}$ & Protein & $\begin{array}{l}\text { Anorg. } \\
\text { Anteil }\end{array}$ & $\begin{array}{c}\text { Menge } \\
\text { Trocken-Gew. }\end{array}$ & $\begin{array}{l}\text { Kohlen- } \\
\text { hydrate }\end{array}$ & Summe \\
\hline A & $\begin{array}{c}7,7-11,3 \\
10,2\end{array}$ & $1,6-3,5,2$ & $14,0-17,4$ & $13,2-17,7,9$ & $9,5-13,9$ & $1,7-3,0$ & $35-44$ & $.7,7-11,8$ & $\underset{170}{150-310}$ & 40,5 & 89,1 \\
\hline B & $8,7-12,8$ & $\underset{2,9}{2,2-3,3}$ & $\frac{15,0-21,8}{16,6}$ & $14,6 \underset{16,5}{-19,2}$ & $12,5-15,1$ & $2,4-3,8$ & $36-45$ & $8,3-11,9$ &.$\underset{\text { von A }}{65-95 \%}$ & 44,8 & 96,3 \\
\hline C & $10,9-13,0$ & $2,7-3,4$ & $\underset{21,4}{19,5-23,7}$ & $\begin{array}{c}17,2-21,5 \\
20,0\end{array}$ & $12,8-15,9$ & $2,4-3,8$ & $37-46$ & $4,3-8,8$ & $\underset{\text { von B }}{85-95 \%}$ & 51,4 & 97,4 \\
\hline
\end{tabular}

Tab. 2

Literaturübersicht der Proteinausscheidung in Normalurin

\begin{tabular}{|c|c|c|c|c|}
\hline Autor & Jahr & Methode & Bereich & \\
\hline $\begin{array}{l}\text { MÖRNER (1) } \\
\text { RIGAS und Mitarbeiter (52) } \\
\text { BOYCE und Mitarbeiter (61) } \\
\text { GRANT und Mitarbeiter (12) } \\
\text { BOYCE und Mitarbeiter (62) } \\
\text { KEUTEL und Mitarbeiter (63) } \\
\text { ANDERSON und Mitarbeiter (64) } \\
\text { BOURRILLON und Mitarbeiter (54) } \\
\text { TIDSTRøM (50) } \\
\text { GRIEBLE und Mitarbeiter (20) } \\
\text { KEUTEL (65) } \\
\text { JøRGENSEN und Mitarbeiter (66) } \\
\text { HEMMINGSON und Mitarbeiter (53) } \\
\text { HEMMINGSON und Mitarbeiter (53) } \\
\text { HEMMINGSON und Mitarbeiter (53) } \\
\text { GRÄSSLIN und Mitarbeiter }\end{array}$ & $\begin{array}{l}1895 \\
1951 \\
1954 \\
1957 \\
1958 \\
1959 \\
1960 \\
1960 \\
1963 \\
1965 \\
1965 \\
1967 \\
1967 \\
1967 \\
1967 \\
1969\end{array}$ & $\begin{array}{l}\text { Dialyse, Trocknung } \\
\text { Berechnet über Elektrophorese } \\
\text { Dialyse, Ultrafiltration, Lyophilisation } \\
\text { Filtration, N-Analyse } \\
\text { Ultrafiltration, Lyophilisation } \\
\text { Ultrafiltration, Trocknung } \\
\text { Ultrafiltration, Dialyse, Lyophilisation } \\
\text { Dialyse, Lyophilisation } \\
\text { Kolorimetrie } \\
\text { Dialyse, Biuret-Methode } \\
\text { Dialyse, Lyophilisation } \\
\text { Gelfiltration, Kolorimetrie } \\
\text { Dialyse, Kolorimetrie } \\
\text { Dialyse, Kolorimetrie } \\
\text { Dialyse, N-Analyse } \\
\text { Druckfiltration, Dialyse, Lyophilisation }\end{array}$ & $\begin{array}{l}22-78 \\
30-50 \\
87-97 \\
46-66 \\
\left.505^{*}\right) \\
\left.175^{*}\right) \\
\left.200^{*}\right) \\
120-150 * *) \\
16-54 \\
\left.201^{*}\right) \\
474 *) \\
82-156 \\
38-394 \\
38-394 \\
2-190 \\
150-310\end{array}$ & $\begin{array}{l}\mathrm{mg} / \mathrm{l} \\
\mathrm{mg} / 24 \mathrm{Stdn} . \\
\mathrm{mg} / 24 \text { Stdn. } \\
\mathrm{mg} / 24 \text { Stdn. } \\
\mathrm{mg} / 24 \text { Stdn. } \\
\mathrm{mg} / \mathrm{l} \\
\mathrm{mg} / \mathrm{l} \\
\mathrm{mg} / \mathrm{l} \\
\mathrm{mg} / 24 \mathrm{Stdn} . \\
\mathrm{mg} / \mathrm{l} \\
\mathrm{mg} / 24 \mathrm{Stdn} . \\
\mathrm{mg} / 24 \text { Stdn. } \\
\mathrm{mg} / 24 \text { Stdn. } \\
\mathrm{mg} / 24 \text { Stdn. } \\
\mathrm{mg} / 24 \text { Stdn. } \\
\mathrm{mg} / 24 . S t d n .\end{array}$ \\
\hline
\end{tabular}

*) Mittelwert. **) Bei Kindern von 6-12 Jahren.

\section{Zucker-Test Urin-Glykopro- Zucker-Test tein, hydrolysiert}

1 2 3

\section{Glucurono- \\ lacton}
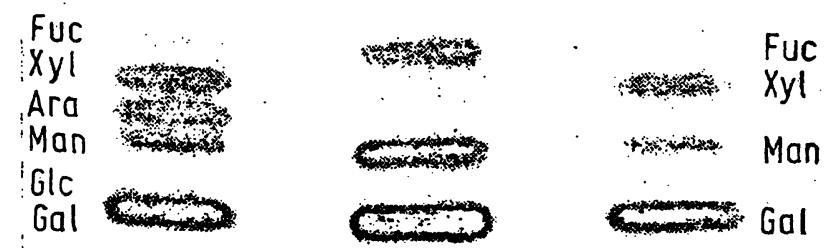

Abb. 1

Dünnschichtchromatographie von Neutralzuckern auf Cellulose-Platten $1=$ Testlauf $2=$ Substanz A aus Normalurin, Hydrolyse mit $\mathrm{H}_{2} \mathrm{SO}$.
$3=$ Testlauf

$\alpha_{1}$-saures Serumglykoprotein, die langsam wandernde Fraktion durch Vergleich mit immunologisch reinen Referenzpräparaten (Behring - Werke, Marburg) als Transferrin identifiziert werden.

Substanz $C$ zeigt eine starke Abnahme von Albumin und eine Zunahme von $\alpha_{1}$-saurem Glykoprotein. G 4 wurde über die PAS-Reaktion angefärbt und zeigt lediglich $2-3$ schwache Glykoproteinbanden. In den Gelzy-
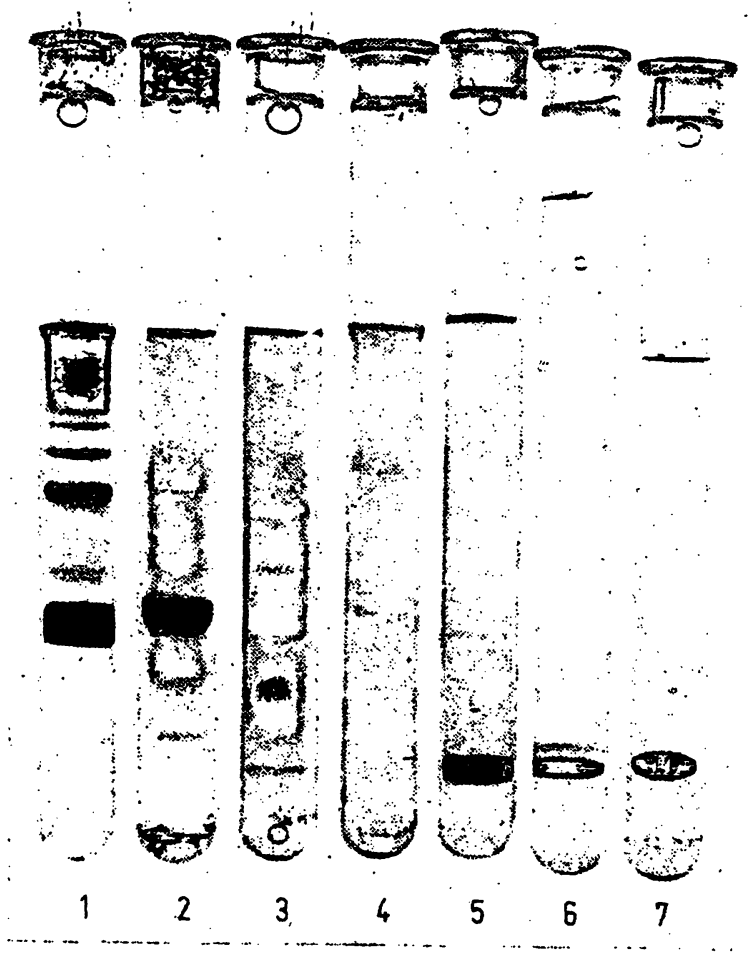

Abb. 2

Disc-Elektrophorese, 7,5proz. Gel

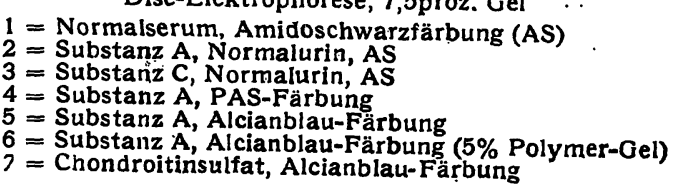

lindern $G 5$ und $G \sigma$ konnte nach spezifischer Anfärbung mit Alcianblau ein Chondroitinsulfat-Gemisch durch Vergleich mit authentischen Material (G 7) identifiziert werden.

Die Glykosaminoglykane des Normalurins ließen sich in 5proz. Polyacrylamidgel (G G) in zwei Fraktionen 


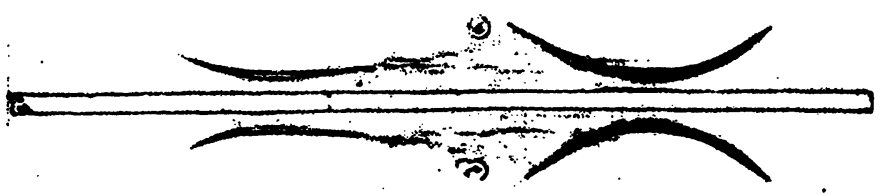

Abb. 3

Immunoelektrophorese: nichtdialysierbarer Urinanteil gegen Antihumanserum

auftrennen. Für die anderen Elektrophoresen wurde 7,5proz. Mittelporgel benutzt. Abbildung 3 zeigt die Immunoelektrophorese der nichtdialysierbaren Urinfraktion (Substanz A) gegen polyvalentes Antihumanserum.

\section{Diskussion}

Wir bestimmten den Wert für die physiologische Proteinurie zu $150-310 \mathrm{mg} / 24 \mathrm{Stdn}$. Bei $85 \%$ der untersuchten Normalpersonen lag die Ausscheidungsrate im relativ engen Bereich von $150-200 \mathrm{mg} / 24 \mathrm{Stdn}$.

Tabelle 2 zeigt eine Literaturübersicht des nichtdialysierbaren bzw. nichtultrafiltrierbaren Anteils in Normalurin. Die Vergleichswerte der verschiedenen Autoren variieren erheblich und zweifellos sind diese Unterschiede auch von der angewandten Methode abhängig. Hierbei ist noch zu berücksichtigen, daß bei den niedrigen Werten meist mit den entsprechenden $\mathrm{Me}-$ thoden lediglich der Proteinanteil in Lösung bestimmt wurde.

Tidstrøm (50) und Mclagan (51) erhielten bei Männern höhere Ausscheidungsraten als bei Frauen. Wir konnten diesen Befund nicht bestätigen und fanden in Übereinstimmung mit RIGAS (52) und HEMmingsoN und Mitarbeitern (53) keine signifikanten Unterschiede in der Menge und den Analysenergebnissen hinsichtlich des Geschlechts. Auch das Alter der Personen (zwischen 20 und 40 Jahren) und die unterschiedlich großen Urin-Mengen/24 Ștdn. spielen nach unseren Untersuchungen keine Rolle.

In Tabelle 1 sind die Analysenergebnisse für die Substanzen A, B und C mit den Bereichen und Mittelwerten zusammengestellt. Der Neuraminsäuregehalt mit 10,2\% und der Gesamtkohlenhydratgehalt mit 40,5\% für Ausgangssubstanz A steigen an über deren 1proz. wäßr. Auszug (Substanz B) mit 11,1\% Neuraminsäure und $44,8 \%$ Gesamtkohlenhydrate bis zur $2 \mathrm{mal}$ dialysierten Substanz C: $11,8 \%$ Neuraminsäure und 51,4\% Gesamtzuckergehalt.

$\mathrm{Zu}$ sehr ähnlichen Analysenergebnissen kamen 1960 BourriLLON und KapLAN (54) bei der Untersuchung des nichtdialysierbaren Anteils des Urins von Kindern im Alter von 6 bis 12 Jahren, mit 9,7\% Neuraminsäure und $40 \%$ Kohlenhydratanteil.

Bei Substanz A wurde für Hexuronsäuren ein Mịttelwert von $3,0 \%$ gefunden. Hierbei ist die Beeinflussung der Chromogenbildung durch Neutralzucker bei der Carbazol-Methode nach Dische (43) über einen Korrekturfaktor berücksichtigt worden. Der Anteil an Glucuron- säure in Chondroitinsulfaten liegt wie bekannt bei $33 \%$. Hiernach läßt sich bei Substanz A aus dem Hexuronsäuregehalt ein Chondroitinsulfatanteil von $10 \%$ berechnen. Mit Hilfe der Disk-Elektrophorese konnte dieser Wert bei quantitativen Auftrag und Anfärbung mit Alcianblau nach semi-quantitativen Konzentrationsvergleich mit reinem Chondroitinsulfat bestätigt werden.

Es ist bekannt, daß die Glycosaminoglycan-ProteinKomplexe Xylose enthalten und es ist daher zu diskutieren, ob die im nichtdialysierbaren Anteil von Normalurin von uns (55) und anderen Autoren (56 bis 58) beschriebene Xylose ausschließlich aus Mukopolysaccharid-Protein-Komplexen stammt.

Ebenso wie der Mukopolysaccharidanteil wurde von uns im Dialyserückstand des Urins auch der mittlere Gehalt an Albumin mit Hilfe der Disk-Elektrophorese bestimmt. Nach Anfärbung mit Amidoschwarz wurde durch optischen Konzentrationsvergleich ein Albuminwert um $10 \mathrm{mg} / 24 \mathrm{Stdn}$. ermittelt. Dieser Befund stimmt gut mit dem von Poortmans und Jeanloz (59) gefundenen Wert von $12 \mathrm{mg}$ für die Albuminausscheidung in 24 Stdn. überein. Die Autoren hatten mit Hilfe der Radial-Immundiffusion die ausgeschiedenen Plasmaproteine für 12 verschiedene Spezies quantitativ bestimmt. Danach werden pro Tag insgesamt $17,5 \mathrm{mg}$ Serumproteine im Urin ausgeschieden. Legt man diese Ausscheidungsrate unseren Untersuchungen zugrunde, so besteht der gesamte Dialyse-Rückstand des Normalurins im Mittel zu 10\% aus Plasmaproteinen, wobei allein $7 \%$ auf Albumin entfallen. Der Anteil an anorganischen Bestandteilen im Dialyserückstand wurde gravimetrisch bestimmt und liegt im Mittel bei 9,6\% (s. Tab. 1). Während in Normalurin die molaren Verhältnisse der Mittelwerte von $\mathrm{Na}^{+}: \mathrm{K}^{+}: \mathrm{Ca}^{++}$bei 26:10:2 liegen (60), ändert sich ihr Verhältnis im nichtdialysierbaten Anteil (Substanz A) zu 26:1:17, nach flammenphotometrischer Bestimmung.

Die nichtdialysierbaren Urinanteile setzen sich danach aus den in Abbildung 4 prozentual dargestellten Komponenten zusammen.

Wir danken Fräulein S. Neubert und Fräulein G. Sommer für wertvolle Mitarbeit bei allen durchgeführten Untersuchungen.



Abb. 4

Zusammensetzung des nichtdialysierbaren Antells in Normalurin (Substanz A) 


\section{Literatur}

1. Mörner, K. A. H., Scand. Arch. Physiol. 6, 332 (1895). 2. Berggi̊rd, J., Ark. Kemi 18, 291 (1961). - 3. TAmm, J. und F. L. Horsfali, JR., J. Expol. Med. 95, 71 (1952). - 4. Hemmingson, L. undF. Skov, Clin. chim. Acta (Amsterdam) 19, 81 (1918). 5. Tamm, J. und F. L. Horsfall, JR., Proc. Soc. exp. Biol. Med. 74, 168 (1950). - 6. King, J. S., M. L. Frelden, H. O. GoodMaN und W. H. Boyce, Arch. Biochem. Biophysics 49, 310 (1961). 7. MAXField, M., Biochem. biophysica Acta (Amsterdam) 49, 548 (1961). - 8. Maxfredd, M., Arch. Biochem. Biophysics 89, 281 (1960). - 9. di Ferrante, N. und C. Rich, J. Laborat. Clin. Med. S. Louis 48, 491 (1956). - 10. Hamerman, D., F. T. Hatch, A. REIFE und K. W. BARTZ, J. Laborat. Clin. Med. S. Louis 46, 848 (1955). - 11. Krng, J. S., JR., W. H. Boyce, J. M. LitrtLe und C. Artom, J. Clin. Invest. 37, 315 (1958). - 12. Grant, G. H. und P. H. Everall, J. Clin. Path., London 10, 360 (1957). - 13. Patte, J. C., G. BALdASSAIRE und J.Loret, Rev. franç. étud. clin. biol. 111, 960 (1958). - 14. Biserte, G., A. Breton und R. Havez, Arch. franç. pédiatr. 15, 5 (1959). - 15. Grant, G. H., J. Clin. Path., London 12, 510 (1959). - 16. Heremans, M. T., J. P. Vaerman und J. F. Heremans, in: Peeters, H., editor, Protides of Biol. Fluids, Proc. 7th Colloq., Bruges (1959), Elsevier Publ. Co. (1960). - 17. de Vaux-Saint-Cyr, C., und J. C. Patte in Grabar, P. und P. BurTIN, editors: L'analyse Immunoelectrophoretique, Masson et Cie., Paris (1960). - 18. Bourrillon, R. und J. C. Kaplan, Clin. chim. Acta (Amsterdam) 5, 732 (1960). - 19. BergGARD, I., Nature (London) 187, 776 (1960). - 20. GrIEBLE, H. G., J. Courcon und P. Grabar, J., Laborat. Clin. Med. S. L ouis 66, 216 (1965). - 21. AdDrs, T., Harvey Lectures Ser. 23, 222 (192728). - 22. Kobayasi, T., J. Biochem. Tokoy 28, 31 (1938). 23. Astrup, P., Acta pharmacatox., K'hvn 3, 165 (1947). - 24. Kerby, G. P., J. Clin. Invest. 33, 1168 (1954) - - 25. Di Ferrante, N. und C. RrCH, Clin. chim. Acta 1, 519 (1956). - 26. Di Ferrante, N., J. Laborat. Clin. Med., S. Louis 61, 633 (1963). - 27. HereMANS, J. F., J. P. VAERMan und M. T. Herenrans Nature (London) 183, 1606 (1959). - 28. Loewr, G., Ann. Rheumat. Dis., London 18, 239 (1959). - 29. Teller, W. M., E. C. Burke, J. W. Rosevedr und B. T. McKenzie, J. Laborat. Clin. Med., S. Louis 59, 95 (1962). - 30. BRUNish, R. und B. SöRENSEN, Dermatologica 130,165 (1965). - 31. TelleR, W., Mschr. Kinderheilk. 112, 538 (1964). 32. DelbrücK, A., H. Grimme und K. Wette, diese Z. 1,10 (1967). - 33. MaUrer, H. R., Disk-Elektrophorese, Walter de
Gruyter \& Co., Berlin (1968). - 34. Davrs, B. J., Preprint „Disc Elektrophoresis" Distillation Prod. Div. Eastman Kodak Co., Rochester (1962). - 35. Caldwell, R. C. und W. Prgman, Arch. Biochem. Biophysica 110, 91 (1965). - 36. KAO, K. Y. T., J. G. Leslie und T. H. McGavack, Proc. Soc. exp. Biol. Med. 122, 1129 (1966). - 36a. Scheidegger, J. J., Internat. Arch. Allergy 7, 103 (1955). - 37. GrässLIN, D. und H. WeICKER, Clin. chim. Acta (Amsterdam) 21, 15 (1968). - 38. SvenNerholm, 'L., Biochim. biophysics Acta (Amsterdam) 24, 604 (1957). - 39. WARren, L., J. biol. Chemistry 234, 1971 (1959). - 40. YEMM, E. W. und A. J. Wirlis, Biochem. J. 57, 508 (1954). - 41. WinzLeR, R. J. in D. Glick: Methods Biochem. Analysis 2, 279 (1955). - 42. Dische, Z. und L. B. Shetrles, J. biol. Chemistry 175, 595 (1948). - 43. Dische, Z., J. biol. Chemistry 167, 189 (1947). - 44. Gregory, J. D., Arch. Biochem. Biophysics 89, 157 (1960). - 45. Cessr, C. und F. Prírego, Biochem. J. 77, 508 (1960). -46. Bradstreet, R. B., Analytic. Chem. 26, 185 (1954). 47. Svennerholm, L. Acta Soc. Med. Upsal. 61, 75 (1965). 48. Berggard, I., Ark. Kemi 18, 315 (1961). - 49. Wallenfels, K., E. Bernt und G. Limberg, Angew. Chem. 23, 581 (1953). 50. TrpSTR øM, B., Scand. J. Clin. Laborât. Invest. 15, 167 (1963). 51. Anderson, A. J. und N. F. McLagan, Biochem. J. 59, 638 (1955). - 52. Rigas, D. A. und C. G. HeLLER, J. Clin. Invest. 30, 853 (1951). - 53. Hemmingson, L. und F. Skov, Clin. chim. Acta 19, 81 (Amsterdam) (1968). - 54. Bourrilion, R. und J. C. Kaplan, Clin. chim. Acta (Amsterdam) 5, 732 (1960). - 55. WeICKER, H. und D. Grässtin, Nature (Ĺondon) 212, 715 (1966): - 56. Gotrschalk, A., Nature (London) 170, 662 (1952). 57. Montreuri, J., St. Jans Hosp., Brügger (Belg.), $3^{e}$ Colloq., (1955). - 58. Tominaga, F., K. Oka und H. Yoshida, J. Biochem. Tokyo 57, 717 (1965). - 59. Poortmans, J. und R. W. Jeanloz, J. Clin. Inviest. 47, 386 (1968). - 60. Documenta Gergy, Wissenschaftl. Tab., 7. Auflage (1968). - 61. BoyCE, W. H., F. K. Garvey und C. M. NorfleEt, J. Clin. Invest. 33, 1287 (1954). - 62. BoYCE, W. H., J. S. King, Jr., Lirrue und C. J. Artom, J. Clin. Invest. 37, 1658 (1968). - 63. Keutel, H. J., Clin. chim. Acta (Amsterdam) 11, 341 (1965). - 64. ANDERson, A. J., M. H. LEPPER und R. J. Winzer, Biochem. J. 77, 581 (1960). - 65. Keuter, H. J., G. Herrmann und W. Licht, Clin. chim. Acta (Amsterdam) 4, 665 (1959). - 66. JørGeNSEN, M. B., Acta med. Scand. 181, 153 (1967).
Dr. D. Grässlin

2 Hamburg 20

Martinistr. 52

Universitäts-Frauenklinik 


\title{
BULLETIN \\ DE LA \\ SOCIÉTÉ DE CHIMIE BIOLOGIQUE \\ („Berichte der Gesellschaft für biologische Chemie“) \\ Unter Mitwirkung des \\ „CENTRE NATIONAL DE LA RECHERCHE SCIENTIFIQUE“ \\ (National-Centrum für wissenschaftliche Forschung) veröffentlicht
}

R. PERLĖS

Hilfs-Generalsekretär

\section{J. E. COURTOIS}

Generalsekretär
Y. RAOUL

Hauptredakteur

Sekretariat und Redaktion

4, avenue de l'Observatoire, Paris (6\%)

Herausgeber:

Masson et Cre, 120, Boulevard Saint-Germain, Paris (6e)

Der „Bulletin de la Socí́té de Chimie Biologique“ veröffentlicht jährlich 11 Hefte; diese enthalten die Arbeiten der französischen Biochemiker, welche der „Socrété DE CHImIE Brologrque“ (Gesellschaft für biologische Chemie) angehören.

\author{
Abonnementspreis 1969: \\ Frankreich und „Franc-Zone“ . . . 150 francs \\ Belgien . . . . . . . . . 1684 belges \\ Andere Länder . . . . . . . 165 francs
}

Aus unserem Elektrodenprogramm:

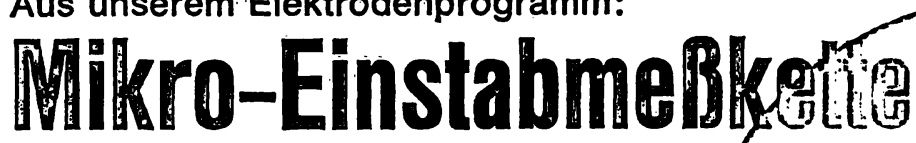

Mro-Elnstabmentyole

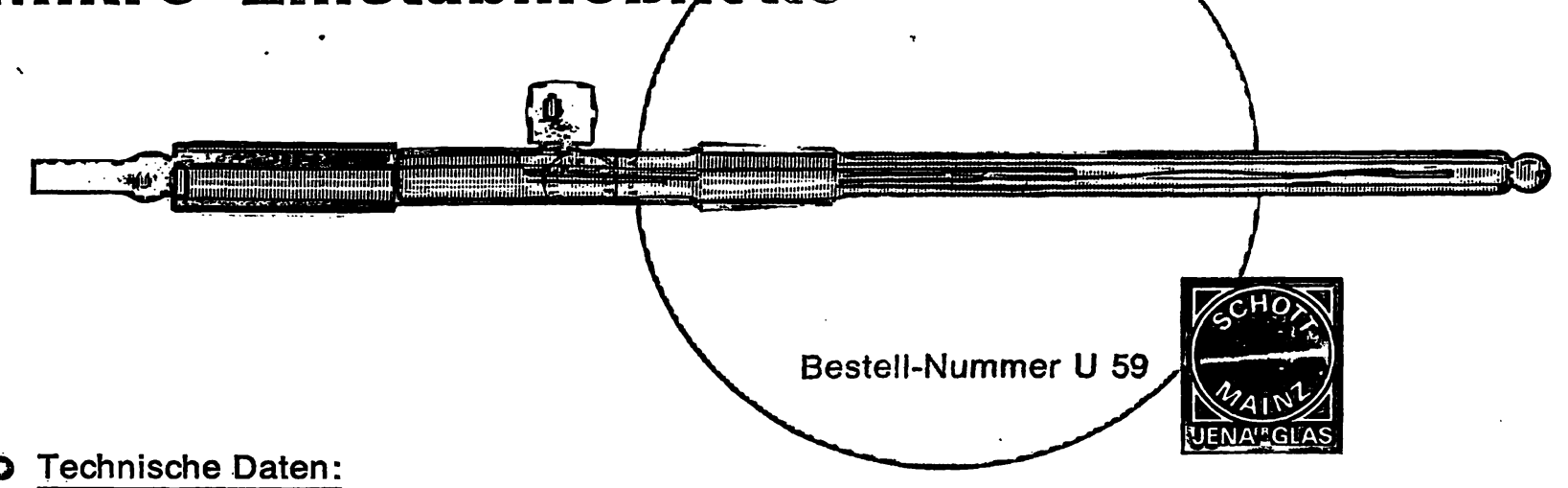

- Einsatzbereich $\mathrm{pH} 0 \ldots 14,10 \ldots 80^{\circ} \mathrm{C}$ O Silberchlorid-Ableitung $O$ Nullpunkt $=\mathrm{pH} 7,0$

- Membranwiderstand $400 \mathrm{M} \Omega$ bei $25^{\circ} \mathrm{C} O$ Platindiaphragma $O$ Einbauschliff NS $7,5 / 16$

- Länge ab Schliffunterkante $80 \mathrm{~mm} \odot$ Schaftdurchmesser $5 \mathrm{~mm} \quad 0 \quad$ Kabellänge $1 \mathrm{~m}$

- Anschlußstecker nach Wahl

- Lieferung über den Fachhandel oder ab Schnelldienstlager Mainz, Tel. 6061, FS 04187849

\section{SCHOTT | JENAER GLASWERK SCHOTT \& GEN., MAINZ}

Wir stellen aus: ANALYTICA $1970 \cdot$ Halle $5 \cdot$ Stand 5219 


\section{Hoppe-Seyler's Zeitschrift für Physiologische Chemie}

Nachdruck Band 1-282 (1877-1945) zuzüglich 8 Registerbände

Subskriptionspreis broschierte komplette Serie DM 15 600,-

Zur Komplettierung unvollständiger Serien werden nachstehend genannte Einzelbände zur Subskription gestellt. Subskriptionspreis broschiert je DM 70,-:

$\begin{array}{rrrll}2 & 17 & 75 & 177 & 269 \\ 6 & 18 & 81 & 182 & 270 \\ 8 & 20 & 82 & 203 & 271 \\ 10 & 23 & 92 & 211 & 275 \\ 11 & 31 & 132 & 212 & 276 \\ 12 & 33 & 141 & 218 & 280 \\ 13 & 34 & 175 & 219 & \text { Reg. Bd. } \\ 15 & 69 & 176 & 235 & 226-250\end{array}$

Der Nacbdruck kann erst vorgenommen werden, wenn eine ausreichende Anzabl von Subskriptionen vorliegt. Alle hier nicht aufgeführten Bände der Serie sind noch in geringer Anzahl lieferbar. Der Ladenpreis für den broschierten Einzelband beträgt DM 85,-

Walter de Gruyter \& Co • Berlin 